\title{
EDITORIAL
}

\section{Can we classify central serous chorioretinopathy better? Yes we}

\section{can}

(C) The Author(s), under exclusive licence to The Royal College of Ophthalmologists 2021

Eye (2022) 36:487; https://doi.org/10.1038/s41433-021-01786-6

In the journal this month, Arora et al. present their correlation of visual acuity outcomes with a novel multimodal imaging based classification of central serous chorioretinopathy (CSCR) [1]. CSCR is an enigmatic but relatively common cause of visual impairment. Vision in CSCR is usually reduced due to the presence of subretinal fluid (SRF). Spontaneous resolution, if it is going to occur, typically does so within 3 months of onset [2]. So, patients with persistent or recurring subretinal fluid beyond 3 months may be defined as chronic CSCR. Each year there are 10 new cases per 100,000 men and 2 cases per 100,000 women in the population [1]. The cause is unknown although it can occur in families and several genetic determinants have been associated with $\operatorname{CSCR}[2,3]$. It is not a benign disease with moderate vision loss in circa $30 \%$ and some patients having visual impairment to $6 / 60$ or worse [4]. The field is also lacking robust placebo controlled randomised clinical trials, thus making it difficult to decide if treatments are effective or not. For example, eplerenone is a commonly prescribed therapy for CSCR, however a recent placebo controlled RCT has showed it is completely ineffective [5]!

A PUBMED search shows there are over 2771 peer reviewed articles on CSCR. However, what do we mean by CSCR? If we do not define the disease consistently, it is extremely difficult to aggregate the results of different papers as you might be comparing apples with oranges e.g. different authors have different definitions of what "chronic" CSCR is. Some authors describe chronic CSCR as SRF being present for more than 3 months while others define chronicity as SRF being present for more than 6 months etc. Therefore, it is important to adopt a common classification system. This will aid evaluation of the prognosis of different sub-types of CSCR and also allow comparison of potential treatments in meta-analyses. To this end, recently a group of experts met and proposed a novel classification system for CSCR [6]. The classification is relatively straightforward to apply to cases (see Table 1 in Arora et al.'s article [1]) which should allow its widespread adoption in future clinical reports of CSCR.

However, is it useful? In this report the authors evaluated the usefulness of this classification system in 229 eyes of 213 patients. They found that older age of patients, foveal involvement and outer retinal atrophy were all factors associated with a poor visual acuity at presentation. Eyes defined as complex or atypical CSCR also showed a significant trend towards severe visual loss. Complex cases also had more recurrent episodes and more persistent SRF. Interestingly the prevalence of macular neovascularization was the same between simple and chronic cases. This paper is important as it shows the classification system can usefully correlate structure with function.

In summary, applying the outcomes from this paper to new patients with CSCR and applying the novel classification system used here will allow clinicians to provide better prognostic information to their patients on the likely outcome of their disease.

More work is needed as the authors correctly highlight. For example, the usefulness of this classification in predicting treatment response. Nevertheless, it is a useful start, showing that function can correlate with this structural classification system. If more studies use this classification system, we will be able to gain further insights into this disease by aggregating results from different studies.

\author{
Andrew Lotery $\mathrm{DD}^{1 \text { 网 }}$ \\ ${ }^{1}$ Professor of Ophthalmology, University of Southampton, \\ Southampton, UK. ${ }^{\bowtie e m a i l: ~ a . j . l o t e r y @ s o t o n . a c . u k ~}$
}

\section{REFERENCES}

1. Arora S, Maltsev DS, Sahoo NK, Parameshwarappa DC, lovino C, Arora T, et al. Visual acuity correlates with multimodal imaging-based categories of central serous chorioretinopathy. Eye. 2021 (in press).

2. Gemenetzi M, De Salvo G, Lotery AJ. Central serous chorioretinopathy: an update on pathogenesis and treatment. Eye. 2010;24:1743-56.

3. Kaye R, Chandra S, Sheth J, Boon CJF, Sivaprasad S, Lotery A. Central serous chorioretinopathy: an update on risk factors, pathophysiology and imaging modalities. Prog Retin Eye Res. 2020;79:100865.

4. Loo RH, Scott IU, Flynn HW Jr, Gass JDM, Murray TG, Lewis ML, et al. Factors associated with reduced visual acuity during long-term follow-up of patients with idiopathic central serous chorioretinopathy. Retina. 2002;22:19-24.

5. Lotery A, Sivaprasad S, O'Connell A, Harris RA, Culliford L, Ellis L, et al. Eplerenone for chronic central serous chorioretinopathy in patients with active, previously untreated disease for more than 4 months $(\mathrm{VICl})$ : a randomised, double-blind, placebo-controlled trial. Lancet. 2020;395:294-303.

6. Chhablani J, Cohen FB. Multimodal imaging-based central serous chorioretinopathy classification. Ophthalmol Retin. 2020;4:1043-6.

\section{AUTHOR CONTRIBUTIONS}

$\mathrm{AL}$ conceived of and wrote this editorial.

\section{COMPETING INTERESTS}

AL has participated in international discussions on CSCR classification which has included some of the authors of the related paper [1].

\section{ADDITIONAL INFORMATION}

Correspondence and requests for materials should be addressed to Andrew Lotery. Reprints and permission information is available at http://www.nature.com/ reprints

Publisher's note Springer Nature remains neutral with regard to jurisdictional claims in published maps and institutional affiliations. 